\title{
Luigi Mancinelli, Giulio Ricordi y Francesco Tamagno, figuras fundamentales en la recepción del primer Puccini en España: el estreno de Edgar en el Teatro Real $(1892)^{1}$
}

\author{
Luigi Mancinelli, Giulio Ricordi and Francesco \\ Tamagno, Fundamental Figures in the Reception \\ of Early Puccini in Spain: The Premiere \\ of Edgar at the Teatro Real (1892)
}

Giacomo Puccini (1858-1924) solo estuvo una vez en España. Fue durante los meses de febrero a marzo de 1892, en los que viajó desde Italia a Madrid para supervisar los ensayos de Edgar y asistir a su posterior estreno en el Teatro Real. A diferencia de Giuseppe Verdi años antes, Puccini apenas tuvo tiempo para conocer otras ciudades españolas. Los ensayos absorbieron todo su tiempo y, tras el estreno, rápidamente regresó a Milán. Edgar fue la primera ópera de Puccini que consiguió estrenarse fuera de Italia, de ahí su importancia para entender la recepción del músico en el extranjero y, sobre todo, en España. Por todo ello, en este artículo pretendemos estudiar el estreno de la primera ópera de Puccini en España, la recepción de la obra y del compositor italiano en Madrid, así como la estancia del músico en la mencionada ciudad.

Palabras clave: estreno operístico, Giacomo Puccini, Edgar (ópera), Teatro Real (Madrid), relaciones musicales España-Italia, recepción operística, producción operística.

Giacomo Puccini (1858-1924) only travelled to Spain once. This trip took place during the months of February and March 1892, when he came to Madrid to supervise the rehearsals of Edgar and attend its subsequent premiere at the Teatro Real. Unlike Giuseppe Verdi years earlier, Puccini barely had time to visit other Spanish cities. The rehearsals took up all of his time and, after the premiere, he quickly returned to Milan. Edgar was Puccini's first opera to be premiered outside Italy, hence its importance for understanding the composer's reception abroad and particularly in Spain. This article thus seeks to examine the premiere of Puccini's first opera in Spain, the reception of the work and the Italian composer in Madrid, as well as his visit to the city.

Keywords: opera premiere, Giacomo Puccini, Edgar (opera), Teatro Real (Madrid), musical relations between Spain-Italy, opera reception, opera production.

${ }^{1}$ Los resultados aquí presentados forman parte de mi tesis doctoral en curso, que versa sobre la recepción de Giacomo Puccini en España (1890-1925). 


\section{Introducción}

Edgar (1889) fue la primera ópera de Giacomo Puccini que se estrenó en España. Hasta ese momento, el italiano era un completo desconocido en el país. El músico viajó desde Milán a Madrid para supervisar los ensayos y asistir al estreno. La estancia de Puccini en la capital y la recepción de Edgar en la misma es un tema que ha sido tratado de manera general por autores como José Subirá ${ }^{2}$ o Federico Oliván ${ }^{3}$. Los trabajos más recientes que han estudiado la recepción del músico en España son el artículo escrito por la musicóloga Diana Díaz González, y la monografia de Andrés Ruiz Tarazona ${ }^{4}$. En el primer caso, la autora fija como marco de estudio el estreno de Edgar en el Teatro Real hasta el estreno de Madama Butterfly en1907 en ese mismo escenario. La importancia de este trabajo reside en la recepción del italiano bajo el punto de vista de la crítica wagneriana y antipuccinista, un factor fundamental para comprender la visión que se tenía, y sobre todo se difundía, sobre el músico y su obra en la capital española. Ruiz Tarazona, por su parte, realiza un ensayo sobre la relación que algunos compositores como Haydn, Boccherini, Mozart o Beethoven establecieron con el país. En el capítulo dedicado a Puccini, el autor ofrece una aproximación a la recepción del músico y sus obras en España.

\section{Los inicios de la producción escénica de Puccini}

El 31 de mayo de 1884, el milanés Teatro dal Verme acogía el estreno de la primera ópera de un joven Giacomo Puccini: LeVilli. El camino hasta llegar allí no fue fácil. Escrita para uno de los concursos de ópera en un solo acto organizado por el editor Edoardo Sonzogno (1836-1920), la obra no llegó a recibir ninguna mención honorífica por parte del jurado por la supuesta ilegible caligrafia musical del italiano ${ }^{6}$. Ferdinando Fontana (1850-1919), libretista de Le Villi, decidido a que la obra se diese a conocer, organizó un concierto privado en el salón de Marco Sala, un mecenas de artistas milaneses y miembro del movimiento literario denominado Scapigliatura -al cual pertenecía el propio Fontana-, para que Puccini hiciera interpretar al piano algunos números de la ópera ${ }^{7}$. Entre los invitados se encontraron otros miembros de dicho movimiento artístico y

\footnotetext{
2 José Subirá: "Puccini y el puccinismo en Madrid (Evocación conmemorativa de un centenario)", Boletín de la Real Academia de Bellas Artes de San Fernando, 7, 1958, pp. 41-68.

${ }^{3}$ Federico Oliván: Puccini. Su vida y su obra, Madrid, Gráficas Uguina, 1949.

${ }^{4}$ Andrés Ruiz Tarazona: "Giacomo Puccini”, España en los grandes músicos, Madrid, Ediciones Siruela, 2018.

${ }^{5}$ Diana Díaz González: "La crítica wagneriana y la recepción de Puccini en Madrid hasta la Primera Guerra Mundial", Música y prensa. Crítica, polémica y propaganda, Enrique Encabo (ed.), Valladolid, Difácil, 2015, pp. 153-182.

${ }^{6}$ Mosco Carner: Puccini: A Critical Biography, Nueva York, Wellesley College Library, 1959, p. 38.

${ }^{7}$ Julian Budden: Puccini, Roma, Carocci, 2007, p. 59.
} 
literario, como Emilio Praga o Arrigo Boito. Este último, interesado por la obra, inició una colecta para que la ópera pudiese ponerse en escena ${ }^{8}$. La acogida de la ópera tras la première fue favorable por parte de la prensa y del público.

A pesar de ello, ni la obra ni su prometedor compositor tuvieron una gran trascendencia en la prensa madrileña. De hecho, la primera noticia documentada, hasta el momento, en la capital española sobre Le Villi data de 1890. La realizó el crítico Antonio Peña y Goñi con motivo del inestable estado en el que se encontraba el arte musical en Italia. GiuseppeVerdi (1813-1901), por su avanzada edad, se suponía que estaba ya retirado del ambiente operístico9 ${ }^{9}$ La prematura muerte de Amilcare Ponchielli (1834-1886), una de las principales figuras del grupo de compositores italianos en aquel momento, y que ninguno de los jóvenes músicos italianos contemporáneos (principalmente Pietro Mascagni, Ruggero Leoncavallo y Umberto Giordano) acabaran de convencer con sus obras a la crítica y al público, dificultaba la continuidad de la tradicional supremacía de la ópera italiana a finales de siglo. Ante este panorama, Peña y Goñi señaló en su crónica que la primera ópera de Puccini había sido recibida en Italia como "un rayo de luz en aquella penumbra" eclipsada por el estreno de Cavalleria rusticana (1890).

No obstante, a pesar de la escasa repercusión de Le Villi en Italia, su estreno despertó el interés del editor Giulio Ricordi (1840-1912), quien encargó a Puccini una obra más ambiciosa: un drama lírico en cuatro actos titulado Edgar $(1889)^{11}$. Para este segundo trabajo, Ricordi y Puccini volvieron a confiar en el libretista Ferdinando Fontana, quien se basó en el drama La Coupe et les Lèvres de Alfred de Musset ${ }^{12}$.

Edgar se estrenó el 21 de abril de 1889 en el Teatro alla Scala de Milán con un reparto destacado dirigido por Franco Faccio: Gregorio Gabrielesco (Edgar), Aurelia Cataneo (Fidelia), Romilda Pantaleoni (Tigrana), y Antonio Magini-Coletti como Frank ${ }^{13}$. A pesar del renombre de los intérpretes, la ópera fue acogida con frialdad por parte del público y de la crítica. El libreto fue acusado de absurdo y la música recibida como demasiado ruidosa por un uso excesivo del metal y la percusión ${ }^{14}$. Giulio Ricordi salió en defensa del libretista y del músico escribiendo la crónica del estreno en su revista La Gazzetta

\footnotetext{
${ }^{8}$ Ibid.

9 Víctor Sánchez: "Manon Lescaut, el primer gran éxito de un instinto operístico", LIX Temporada de Ópera de Oviedo, 2006, s/p.

${ }^{10}$ Ibid.

${ }^{11}$ Ambientada en Flandes en el siglo XIV, el argumento ha sido comparado con el de la Carmen (1875) de Georges Bizet. En este caso, la mujer fatal es la gitana Tigrana, de la que el aldeano Edgar se enamora rechazando a la inocente Fidelia.

${ }^{12}$ Conrad Wilson: Giacomo Puccini, Londres, Phaidon Press, 1997, p. 56.

${ }^{13}$ Michele Girardi: Puccini: His International Art, Londres, Chicago, The University of Chicago, 2000, p. 39.

14 “Edgar. La nuova opera di Puccini”, La Stampa, Turín, 23-4-1889, p. 3.
} 
Musicale di Milano ${ }^{15}$. En ella, Ricordi aceptaba los comentarios recibidos a la vez que intentaba que el público y la crítica diesen una nueva oportunidad a Edgar y a sus autores. Así, el editor italiano explicaba que el libreto no había sido entendido porque Fontana era un "poeta-filósofo"16 y, por lo tanto, las ideas que para él eran claras y convincentes, para el público eran abstractas y no podían seguir con claridad la acción dramática de la obra ni las pasiones de los personajes.

En cuanto al compositor, Ricordi le recomendó públicamente que se tomase las críticas negativas como constructivas y que se sintiera orgulloso de su trabajo, pues "todos los maestros que más tarde hicieron grande su nombre, fueron discutidos agudamente, criticados ferozmente en sus primeras obras"17. Acerca de la música, el editor señaló que Edgar era el fruto de un genio todavía no maduro, y que Puccini poseía cualidades sobresalientes, como el tratamiento melódico ${ }^{18}$. De hecho, esta aptitud innata para la melodía ha sido y es una característica indefectiblemente asociada a la figura del compositor. Debido a esa preponderancia melódica, Ricordi también empezaba a adelantar en su crónica el papel del músico de Lucca como futuro sucesor de Verdi, ya que Puccini era un músico "esencialmente melódico, esencialmente italiano"19.

Edgar fue retirada del cartel de La Scala tras solo dos representaciones y, aunque se habló de la posibilidad de que la ópera abriese la temporada 18901891, dicha propuesta nunca llegó a realizarse ${ }^{20}$.

\section{Las dificiles negociaciones para el estreno de Edgar en Madrid}

Tras haber tomado nota de las críticas italianas, Puccini empezó la revisión de la ópera al mismo tiempo que Ricordi negociaba con Luigi Mancinelli (18481921), director musical en aquel momento del Teatro Real de Madrid (18861893), los últimos detalles para estrenar la obra en la temporada 1890-1891. Luigi Mancinelli fue una figura que influyó muchísimo en las programaciones del Real en aquellos años. Más importante fue su labor como impulsor de la obra wagneriana en Madrid a finales del siglo XIX ${ }^{21}$. El director italiano propició el estreno en el Teatro Real de varios títulos como Tannhäuser (1845) o Los maestros

\footnotetext{
${ }^{15}$ Giulio Ricordi: "Teatro alla Scala. Edgar", La Gazzetta Musicale di Milano, 28-4-1889, p, 271.

${ }^{16}$ Ibid.

17 "Tutti i maestri che poi feccero grande il loro nome, vennero acremente discussi, ferocemente criticati nei loro primi lavori". Ibid. Salvo indicación contraria, todas las traducciones son de la autora.

${ }^{18} \mathrm{Ibid}$.

19 "Essenzialmente melódico, essenzialmente italiano". Ibid., p. 267.

${ }^{20}$ Carta de G. Puccini a Rafaello Franceschini, Milán, 4-5-1889. Giacomo Puccini. Epistolario I (18771896), Gabriella Biagi Ravenni, Dieter Schickling (eds.), Florencia, Edizione Nazionale Delle Opere di Giacomo Puccini, Leo S. Olschki Editore, 2015, p. 100.

${ }^{21}$ José Ignacio Suárez: "La recepción de la obra de Richard Wagner en Madrid entre 1877 y 1893", Cuadernos de Música Iberoamericana, 14, 2005, pp. 102-122.
} 
cantores de Nuremberg $(1862)^{22}$ y de la interpretación de varios fragmentos wagnerianos por la Sociedad de Conciertos ${ }^{23}$, de la cual fue director entre 1891 y 1893. Mancinelli logró imponer el repertorio wagneriano en la capital española a finales de siglo y así reconoció la prensa madrileña su gran labor:

Lo que nunca podremos agradecerle bastante los que adoramos el arte, es la prodigiosa revelación que de la música de Wagner ha realizado [Mancinelli]. Hace poco tiempo Wagner era en Madrid un energúmeno musical, un anarquista del arte [...]. Hoy todo ha cambiado por obra y gracia de Luis Mancinelli y de la Sociedad de Conciertos. Tres meses escasos han bastado para que se efectuara esta transformación saludable que en otros países ha costado años de esfuerzos inauditos [...].Y ahora su obra teatral estará también insensiblemente, poco a poco, en nuestro coliseo de ópera ${ }^{24}$.

Según el epistolario consultado ${ }^{25}$, la idea de representar Edgar en Madrid surgió de manera improvisada después de que Luigi Mancinelli le diese la enhorabuena a Puccini por su ópera y le mostrara su deseo de poder estrenarla en un futuro en el Teatro Real. El compositor italiano, que había conocido al reputado director en 1885 con motivo del estreno de LeVilli en el Teatro Comunale de Bolonia ${ }^{26}$, le agradecía en una carta escrita en octubre de 1886 su consideración de que Edgar pudiese ser interpretada en el teatro madrileño ${ }^{27}$. Más adelante, el músico aprovechó la oportunidad presentada y le propuso formalmente al director italiano llevar a cabo el estreno, tal y como se puede observar en una carta escrita por Puccini a Mancinelli:

Queridísimo Maestro. Sé por Ricordi que la última propuesta que le hice sobre Edgar en Madrid no fue recibida con reticencia sino con gran placer, supe que usted la tomó en cuenta hasta el punto de darme esperanzas. Estaría encantado de poder confiarle mi humilde música porque al menos una vez en la vida me darían la oportunidad de escucharla ${ }^{28}$.

${ }^{22}$ Tannhäuser se estrenó en el Teatro Real el 22 de marzo de 1890. La obertura de Los maestros cantores de Nurenberg pudo escucharse en este escenario dirigida por Mancinelli el 22 de marzo de 1891. El estreno de la ópera completa tuvo lugar el 18 de marzo de 1893. Véase Paloma Ortiz: La recepción de Richard Wagner en Madrid (1900-1914), tesis doctoral, Madrid, Universidad Complutense de Madrid, Departamento de Arte III (contemporáneo), Historia y Ciencias de la Música, 2003, pp. 53-56.

${ }^{23}$ José Ignacio Suárez: La recepción de la obra wagneriana en el Madrid decimonónico, tesis doctoral, Universidad de Oviedo, 2002, p. 98.

${ }^{24}$ F. Bleú: "Madrid Musical. La campaña de la Sociedad de Conciertos. Wagner en Madrid", El Heraldo de Madrid, 14-4-1891, p. 2.

${ }^{25}$ G. Biagi Ravenni, D. Schickling (eds.): Giacomo Puccini. Epistolario I...

${ }^{26}$ El estreno se realizó bajo la dirección de Luigi Mancinelli. Véase Carta de G. Puccini a Luigi Mancinelli, Lucca, 7-1-1885. Giacomo Puccini. Epistolario I..., p. 67.

${ }^{27}$ Carta de G. Puccini a Luigi Mancinelli, Caprino Bergamasco, 17-10-1886. Giacomo Puccini. Epistolario I..., p. 78.

28 "Carissimo Maestro. So da Ricordi che la proposta fattale nell'ultima mia circa Edgar a Madrid non fu raccolta con diniego anzi con mio gran piacere, seppi che lei la prese in considerazione fino a darmi delle speranze. Io sarei felicissimo poter affidarle la mia povera musica perché almeno una volta in vita mia sarebbe dato udirla". Carta de G. Puccini a Luigi Mancinelli, s/l, noviembre de 1887. Giacomo Puccini. 
De este modo, Mancinelli se convertía en el principal contacto del compositor en España y en el mediador entre la empresa del Real y la editorial Ricordi. El proyecto de poner en escena Edgar en Madrid suponía la primera salida al extranjero de la ópera, de ahí que Puccini considerase esta representación española como uno de los negocios más importantes del inicio de su carrera. Así se lo expresó el músico a su hermana Tomaide Puccini:"El invierno que viene espero poder representar Edgar en algún lugar. Tengo pendiente varios negocios entre ellos el de Madrid. Importante" ${ }^{29}$. Al igual que el músico, Ricordi vio en este estreno la oportunidad de enmascarar los fracasos anteriores e impulsar la carrera del joven Puccini. El editor italiano cuidó cada detalle para evitar cualquier contratiempo que afectara el buen éxito de la ópera en la capital española. De hecho, fue tal la obsesión que sintió con este tema, que incluso le llegó a plantear si la presencia de Puccini en Madrid podría afectar su buena acogida:

Te agradezco muchísimo tu preocupación por Edgar. ¡Puccini está incluso conmovido!

Y estaré verdaderamente contento de que se concrete. Ahora, algo totalmente confidencial. En el caso de que Edgar siga adelante, quiero que me digas francamente, amigablemente si te gustaría, o no, que Puccini fuera a Madrid. Dicho de otro modo. No le he dicho nada al respecto, porque él está muy tranquilo, al igual que yo, si te encargan la obra. Tú conoces bien el país, la prensa, la empresa y el público: si la llegada de Puccini es totalmente innecesaria, entonces no digo más ${ }^{30}$.

La ópera apareció anunciada en un folleto publicitario del Teatro Real como una de las obras a representar en la temporada 1890-1891 ${ }^{31}$. Para esa temporada, Edgar compartiría cartel con las "obras de gran repertorio de los maestros Meyerbeer, Wagner, Verdi, Ponchielli y Gluck"32 junto con óperas nuevas como Otello (1887), Simon Boccanegra $(1881)^{33}$, Cavalleria rusticana (1890)

\footnotetext{
Epistolario I..., p. 91.

29 "Questo prossimo inverno spero di dare Edgar in qualche parte. Ho in vista diversi affari fra i quali quello di Madrid. Importante". Carta de G. Puccini a Tomaide Puccini, Milán, 15-1-1890. Giacomo Puccini. Epistolario I..., p. 120.

30 "Ti ringrazio moltissimo della premura che ti dai per l'Edgar. Puccini ne è addiritura commosso! e sarò davvero lietissimo se si combinerà. Ora, una cosa affatto confidenziale. Dato il caso si dia Edgar, desidero che tu mi dica francamente, amichevolmente se ti da caro, o no, che Puccini venga a Madrid. Ben inteso, nulla gli ho detto in proposito, perché tanto egli è tranquillissimo, come lo sono io, se l'opera è affidata a te. Tu conosci bene, paese, stampa, impresa e pubblico: se la venuta affatto superflua la venuta di Puccini, allora sto zitto". Carta de Giulio Ricordi a Luigi Mancinelli, Milán, 23-7-1890. Luigi Mancinelli. Epistolario, Antonio Mariani (ed.), Lucca, LIM, 2000, p. 134.

31 "Folleto informativo o publicitario con la lista completa de la compañía ítalo-española a la que se adjudicaron las representaciones en el Teatro Real de Madrid para la temporada 1890-1891...", Archivo Histórico de la Nobleza [en adelante AHNOB], MICHELENA,C.4,D.73., sn / f. Véase Joaquín Turina Gómez: Historia del Teatro Real, Madrid, Alianza Editorial, 1997, p. 540. En él aparece una relación de todas las óperas representadas en el Teatro Real en la temporada 1890-1891.

${ }^{32}$ Ibid.

${ }^{33}$ Se trataba de la segunda versión definitiva de la obra. La primera versión data de 1857.
} 
y la ópera del compositor español Emilio Serrano (1850-1939), Irene de Otranto (1891). Rápidamente, la prensa madrileña publicaba la información de dicho folleto para anunciar a sus lectores las obras y la compañía que formarían parte de la próxima temporada ${ }^{34}$. Sin embargo, el proyecto de estrenar Edgar no hacía más que retrasarse.

En un primer momento, Mancinelli intentó estrenar la ópera en el otoño de 1890 pero, debido a que las modificaciones y revisiones de Puccini no estuvieron a tiempo, la empresa del Real decidió programar antes otras obras y atrasar el estreno al mes de febrero de $1891^{35}$. Conforme se iba aproximando la fecha, la empresa del teatro y la editorial Ricordi seguían sin cerrar el acuerdo ${ }^{36}$, amenazando así con un nuevo aplazamiento o, lo que sería aún peor, la cancelación del estreno. El editor italiano se puso inmediatamente en contacto con Luigi Mancinelli y con Ramón de Michelena y Padrés (1830-1894), I conde de Michelena y empresario del Teatro Real, para poder llegar a algún acuerdo claro, ya que lo único que habían recibido por parte de la empresa eran falsas esperanzas $^{37}$. Ricordi le transmitió a Mancinelli su malestar expresándole que jamás hubiese podido imaginar que una dirección como la del Teatro Real actuase como una empresa perteneciente a un teatro mediocre ${ }^{38}$. Además, les hizo saber a ambos las consecuencias negativas que supondría aplazar una vez más Edgar. Aparte del disgusto moral que viviría el compositor, en Italia los aplazamientos serían considerados como un rechazo a la ópera, lo que perjudicaría gravemente la prometedora carrera compositiva de Puccini y que Edgar se pudiese representar en otros teatros extranjeros. Si la ópera era rechazada por el teatro más importante de España, después de los continuos fracasos de la obra en el país italiano, pocas empresas estarían dispuestas a programarla. Giulio Ricordi escribió a Michelena:

¡Un retraso sería un verdadero desastre para el pobre Puccini! ¡Conozco bastante bien la prensa italiana que no dudará en decir que todo es una comedia y que la verdad es que la ópera fue rechazada por la Dirección, que no quiere ponerla en escena! Es fácil imaginarse el gravísimo daño que le implicaría al Maestro, además del malestar moral que tendría funestas consecuencias. Todas las esperanzas de Puccini estaban puestas en Madrid. Sé que no hablo con un vulgar empresario sino con un perfecto caballero [...] Le ruego a usted, al Señor Conde, al Maestro

\footnotetext{
34 "La próxima temporada del Real", El Imparcial Madrid, 11-9-1890, p. 4.

${ }^{35}$ Carta de Luigi Mancinelli a G. Puccini, Madrid, 22-11-1890. Luigi Mancinelli. Epistolario..., p. 137.

${ }^{36}$ Carta de Giulio Ricordi a Luigi Mancinelli, Milán, 17-1-1891. Ibid., p. 140.

${ }^{37}$ Ibid., 14-1-1891. Archivio Storico Ricordi (en adelante AR), CLET001985.

${ }^{38}$ Ibid., 17-1-1891. Luigi Mancinelli. Epistolario..., p. 140.
} 
Mancinelli y a los estimados artistas que hagan el esfuerzo de representar este año la obra de Puccini, aunque sea solo 3 o 4 noches [...], yo creo que nadie se arrepentirá de hacerlo por un joven y pobre artista de talento ${ }^{39}$.

En la carta se refleja la incesante apuesta de Ricordi por el joven compositor, lo cual obedecía también a su buen olfato artístico. El editor fue un hombre de negocios con una gran agudeza para intuir y reconocer desde el primer momento, el talento de músicos, libretistas, cantantes o directores de orquesta antes de su fama ${ }^{40}$. Ricordi recibió explicaciones de por qué Edgar no había sido puesta en escena todavía. Mancinelli le comunicó que la falta de tiempo para ensayar debido a la gran cantidad de óperas que debían representar a mitad de temporada, y las presiones sufridas por la empresa para programar las óperas de Wagner y la del compositor español Emilio Serrano, habían sido los motivos de los aplazamientos.

Querido Giulio.

Como habrás comprobado por el telegrama de Michelena y mío, a pesar de mi insistencia y, también hay que reconocerlo, de la buena voluntad de la empresa, no ha sido posible asumir la responsabilidad de una buena ejecución para Edgar [...]. En noviembre, antes de que surgiese la cuestión sobre las obras de Wagner, la empresa estaba totalmente de acuerdo conmigo en representar Edgar antes de fin de año [...]; vinieron después las presiones e insistencias para la obra del maestro Serrano y este conjunto de cosas dio como consecuencia el resultado que más deploro, siendo únicamente mérito mío si la obra de Puccini era aceptada por la empresa y puesta en cartel. Ahora para subsanar, la empresa te enviará una declaración que podrás publicar en todos los periódicos italianos y que explicará las causas por las que Edgar se pospuso al próximo otoño. Por el resto Puccini ya está vengado: la obra de Serrano ha sido un fracaso total ${ }^{41}$.

39 "Un ritardo sarebbe un vero disastro per il povero Puccini! Io conosco assai bene la stampa italiana, la quale non mancherebbe di dire che tutto ciò è una commedia!... e che la verità è che l'opera fu protestata dalla Direzione, che non vuole metterla in scena!! - E' facile immaginarsi il gravissimo danno che ne verrebbe al Maestro, oltre il dispiacere morale che avrebbe funeste conseguenze, tutte le speranze del Puccini essendo riposte con intiera fiducia in Madrid. Io so di parlare non con un volgare impresario, ma con un perfetto gentiluomo [...] Io prego Lei, Signor Conte, il Maestro Mancinelli e gli egregi esecutori a fare il sacrificio di rappresentare quest'anno l'opera del Puccini, fosse pure per 3, o 4 sere soltanto [...] ma io credo che nessuno si pentirà di avere ciò fatto per un giovane e povero artista di talento". Carta de Giulio Ricordi a Ramón de Michelena, Milán, 11-2-1891. AR, CLET001983.

${ }^{40}$ Rut Francia Ferrero: La editorial Ricordi y su aportación a la publicidad italiana de principios del siglo XX, tesis doctoral, Universidad Complutense de Madrid, 2013, p. 25.

41 "Caro Giulio. Come avrai rilevato dal telegramma di Michelena e mio, malgrado la mia insistenza e, bisogna convenirne anche, la buona volontà dell'impresa, non è stato possibile assumere la responsabilità di una buona esecuzione per l'Edgar [...]. Nel novembre, prima che sorgesse la questione per le opere di Wagner, l'impresa era pienamente d'accordo con me di mandar un scena l'Edgar avanti la fine dell'anno [...]; vennero poi le pressioni ed insistenze per l'opera del $\mathrm{M}^{\circ}$ Serrano e questo insieme di cose produsse il risultato che io più di tutti deploro, essendo unicamente merito mio se l'opera di Puccini fu accettata dall'impresa e messa nel cartellone. Ora per riparare, l'impresa ti spedirà una dichiarazione che potrai far pubblicare nei giornali italiani e che spiegherà le cause per le quali l'Edgar dovette essere rimandato al prossimo autunno. Del resto Puccini è già vendicato: l'opera di Serrano ha fatto un fiasco completo". Carta de Luigi Mancinelli a Giulio Ricordi, Madrid, antes del 4 de marzo de 1891. Luigi Mancinelli. Epistolario..., pp. 141-142. 
Lo que más llama la atención de la carta son las palabras de Mancinelli sobre el estreno de la ópera de Emilio Serrano. El director de orquesta parecía alegrarse del fracaso sufrido por el compositor español, ya que como él mismo explicaba a Ricordi, en la decisión de cuál de las dos óperas iría antes a escena se quedó sin el apoyo de la empresa del Real. De modo que, si Edgar se hubiese representado finalmente en esa temporada hubiese sido únicamente gracias a él. La presencia de Mancinelli en el Teatro Real explica la insistencia de Ricordi y Puccini por estrenar Edgar en Madrid y no en otra ciudad de fuera de Italia como, por ejemplo,Viena, París o la propia Barcelona donde existía una gran afición por la ópera italiana. La figura de Mancinelli fue verdaderamente determinante en la recepción del primer Puccini en España. Si él no hubiera sido en aquel momento el director musical del Teatro Real es probable que Edgar no se hubiera representado finalmente, viendo las dificultades presentadas para estrenar la ópera,

Si rastreamos las críticas del estreno de Irene de Otranto (1891), la ópera, aunque recibió numerosos aplausos y valoraciones positivas de parte de la prensa madrileña, no obtuvo un gran éxito ${ }^{42}$. Según la crítica, el público aceptó la ópera sin mostrar mayor entusiasmo. La originalidad del asunto dramático y que el idioma cantado fuese el español, fueron los elementos que despertaron extrañeza entre la audiencia, y señalados como los causantes de esa regular recepción ${ }^{43}$. También se comentó que el público fue al estreno con prejuicios al estar la obra compuesta por un autor español, añadiendo que si la ópera hubiese sido de un músico extranjero su acogida hubiera sido mucho mayor ${ }^{44}$. La producción del músico italiano será, a lo largo de su recepción en el país, juzgada duramente por los defensores de la ópera nacional.

Para evitar futuros enfrentamientos con Ricordi, Mancinelli se ocupó de que los periódicos italianos publicaran el verdadero motivo del aplazamiento de $E d$ gar.Así, el director musical le comunicó a Carlo Brosowich, director del periódico musical Il Trovatore, que se modificara lo publicado respecto a la ópera:

Querido Brosowich. El último número del Trovatore dice: "La obra Edgar de Puccini, prometida en el cartel del Teatro Real de Madrid, se ha esfumado". Como pueden darse varias explicaciones al respecto, te ruego publiques que la obra de Puccini no ha podido estrenarse como se había previsto [...] solo por falta de tiempo. La nueva obra del maestro Serrano necesitó casi dos meses de estudio, tras los cuales el coro no tuvo tiempo de estudiarse Edgar. [...] La empresa ha establecido no retrasar la puesta en escena más allá del próximo diciembre. Te ruego dar acogida a mi carta en tu periódico y te lo agradezco por adelantado ${ }^{45}$.

\footnotetext{
${ }^{42}$ F. Bleú: "Madrid Musical. Irene de Otranto", El Heraldo de Madrid, 18-2-1891, p. 1.

${ }^{43}$ Ibid.

${ }^{44}$ B. Sellav: "Irene de Otranto", La Unión Católica, Madrid, 18-2-1891, p. 2.

45 "Caro Brosowich. L'ultimo numero del Trovatore dice 'LEdgar di Puccini, promesso sul cartellone del Teatro Real di Madrid, è andato in fumo'. Siccome si possono dare varie interpretazioni alla cosa, ti prego di pubblicare che l'opera di Puccini non si è potuta mettere in scena come era disposto [...] unicamente
} 
Finalmente, por falta de tiempo y espacio en la programación, la versión revisada de la ópera no se interpretó en primer lugar en Madrid. Su representación tuvo lugar en la localidad natal del compositor en el Teatro del Giglio el 5 de septiembre de $1891^{46}$. A pesar de ser acogida con notable éxito en Lucca, esta recepción no convenció ni a Ricordi ni al compositor, quienes vieron necesaria otra revisión más. Así, los cuatro actos se comprimieron en tres para que la ópera ganara en concisión y en efecto dramático ${ }^{47}$. Mientras tanto, la prensa madrileña, se hacía pronto eco del éxito en Lucca con motivo de la participación de la soprano española Luisa Gilboni en el papel de Fidelia ${ }^{48}$.

No está claro, a la luz de los documentos localizados, si la nueva revisión en tres actos era la destinada a estrenarse a la primera oportunidad en Madrid. Lo cierto es que se representó antes en el Teatro Comunale de Ferrara (28 de enero de 1892) y en el Teatro Regio de Turín (5 de marzo) ${ }^{49}$. Sobre el estreno en Ferrara y Turín, las críticas italianas volvían a incidir en el desatino del libreto y en el abuso de instrumentos de viento metal y percusión. Aunque el cuarto acto se había fusionado con el tercero y se habían suprimido escenas, el libreto continuaba "pecando" por su sobreabundancia de acción ${ }^{50}$. Mientras, Puccini se revelaba como un músico victorioso que merecía algo más que una simple mención en los periódicos como, por ejemplo, que sus óperas y tendencias fuesen discutidas ${ }^{51}$.

Hasta noviembre de 1891 no comenzaron los primeros ensayos de Edgar en el Teatro Real ${ }^{52}$. Ante la imposibilidad de Puccini de asistir en aquel momento, Mancinelli tuvo que hacer frente él solo a las nuevas correcciones que el músico había añadido tras los estrenos anteriores y a las numerosas instrucciones (tempo, instrumentación, articulación, etc.) que este le hacía llegar a través de la correspondencia ${ }^{53}$. A principios del nuevo año, en enero de 1892, se hacía realidad el deseo por el que tanto habían luchado Ricordi y Puccini: se cerraba el acuerdo con la empresa del Teatro Real para representar Edgar en el cercano mes de mar$\mathrm{zo}^{54}$. Sin embargo, un inesperado contratiempo puso de nuevo en peligro el an-

\footnotetext{
per mancanza di tempo. L'opera nuova del maestro Serrano richiese quasi due mesi di studio, dopo i quali non rimase al corpo corale, il tempo necessario per imparare l'Edgar. [...] Limpresa ha stabilito di non ritardarne la messa in scena oltre il prossimo Decembre. Ti prego di dare nel tuo giornale ospitalità a questa mia e te ne ringrazio anticipatamente". Carta de Luigi Mancinelli a Carlo Brosowich, Madrid, 11-31891. Luigi Mancinelli. Epistolario..., p. 144.

${ }^{46}$ Julian Budden: Puccini: His Life and Works, Oxford, Oxford University Press, 2006, p. 67.

${ }^{47}$ M. Girardi: Puccini: His International Art..., p. 41.

48 "Telegramas", La Correspondencia de España, Madrid, 27-9-1891, p. 1.

${ }^{49}$ M. Girardi: Puccini: His International Art..., p. 42.

${ }^{50}$ Giussepe Depanis: "Arti e scienze. Edgar. Considerazioni generali”, La Stampa, Turín, p. 2.

${ }^{51}$ Ibid., p. 3.

${ }^{52}$ Carta de G. Puccini a Luigi Mancinelli, sn / 1, últimos diez días del mes de noviembre de 1891. Giacomo Puccini. Epistolario I..., pp. 174-175.

${ }^{53} \mathrm{Ibid}$.

${ }^{54}$ Carta de Giulio Ricordi a Francesco Tamagno, Milán, 13-1-1892. AR, CLET001806.
} 
siado estreno. Edgar se quedaba sin protagonista tras la repentina indisposición del tenor Eugenio Durot. Ante esta situación, Puccini se puso en contacto con el tenor Francesco Tamagno (1850-1905) para intentar lo que no pudo conseguir en 1889 para el estreno de La Scala ${ }^{55}$ :que interpretase el papel principal de Edgar. El tenor había cosechado gran fama tras haber protagonizado en 1887 el estreno absoluto de Otello en Milan, de ahí que Puccini quisiera contar con su presencia. Aprovechando que Tamagno había sido contratado para cantar la ópera verdiana en el Teatro Real, el compositor volvió a proponérselo. Tras la indisposición de Durot, Tamagno se convertía en su única salvación:

He sabido que estás por Madrid y tomando mi valor con las dos manos te hago una oración, y te pido un enorme favor que de ser oídos serían una gran ventaja para mi carrera $[\ldots]$.

¡Estábamos cerca del estreno cuando me llegó la noticia de que Durot ha prescindido del contrato! De esta forma mi pobre Edgar se ha quedado sin protagonista y sin esperanza de representación. [...]. Por lo tanto, la propuesta-plegaria que con valor te expongo es que representes la importantísima parte del protagonista. Hace falta valor y audacia ¿eh? pero conociéndote de corazón me he atrevido a hacerte esta propuesta. ¿Se cumplirá? Te confieso que lo espero [...]. ¡ Entre tantas desgracias acaecidas espero al menos ser afortunado en esta ocasión! Te escribo como me dicta el corazón tras tantos tropezones... Es tanta la emoción de pensar que quizás... ¿quién sabe? ¡Tú representarás mi obra! Si lo aceptases te ruego que me escribas dos líneas $[\ldots]$. Es tu decisión, querido amigo $[\ldots]^{56}$.

Puccini puso al tenor en una tesitura muy difícil. El músico presionó a Tamagno para que encarnase el papel protagonista, hasta el punto de dejarle como responsable de que la representación se llevase a cabo finalmente. El cantante no solo tuvo que hacer frente a las súplicas de Puccini sino también a las de Giulio Ricordi. El editor volvió a recurrir al mismo argumento que había utilizado con el conde de Michelena para convencer a Tamagno: su ayuda impulsaría la carrera de un joven artista con talento ${ }^{57}$. Tras la aceptación del

\footnotetext{
${ }^{55}$ Carta de G. Puccini a Francesco Tamago, Milán, 31-12-1888. Giacomo Puccini. Epistolario I..., p. 98.

56 "Ho saputo che tu sei sulle mosse per Madrid ed io prendendo il mio coraggio con due mani ardisco avanzarti una preghiera, chiederti un favore grandissimo che esauditi tornerebbero di immenso vantaggio alla mia carriera [...]. Eravamo dunque vicini all'andata in scena, quando mi giunge notizia che il Durot si è sciolto! - e così il mio povero Edgar è restato senza protagonista e senza speranza di riproduzione! [...] Dunque la proposta-preghiera che coraggiosamente ti espongo è che tu mi abbia a creare la importantissima parte del Protagonista! Ci vuol coraggio e audacia eh? ma pure conoscendoti tanto di buon cuore ho ardito esporti questa idea - Sarò esaudito? ti confesso che lo spero [...]. Fra tante disgrazie capitatemi almeno avessi questa fortuna! Ti scrivo proprio come mi detta il cuore getto giù a sbalzi...è tanta l'emozione a pensare che forse... chi sa? Tu eseguirai roba mia!! Se tu accettassi ti prego scrivermi una riga [...]. Sta in te, caro amico, [...]”. Carta de G. Puccini a Francesco Tamagno, Milán, 11-1-1892. Giacomo Puccini. Epistolario I..., p. 178.

${ }^{57}$ Carta de Giulio Ricordi a Francesco Tamagno, Milán, 13-1-1892. AR, CLET001806.
} 
tenor $^{58}$, el contacto entre el cantante y el músico fue ininterrumpido ante el escaso tiempo de preparación. Puccini tranquilizó a Tamagno haciéndole saber que el papel no supondría ninguna dificultad para él ante su gran capacidad de aprendizaje y por la poca complejidad de la partitura ${ }^{59}$. Además, el tenor había tenido anteriormente la oportunidad de estudiar parte de la obra cuando el músico se la ofreció para el estreno en La Scala ${ }^{60}$. Por otro lado, Puccini continuaba totalmente convencido de que la baja de Durot había sido la mayor de sus fortunas, pues con Tamagno como protagonista el éxito de la ópera estaba asegurado. El autor le agradeció al tenor la decisión de haber aceptado el rol de Edgar ya que, gracias a él, su sueño iba por fin a cumplirse ${ }^{61}$.

La noticia del reemplazo del tenor Eugenio Durot por Tamagno pronto llegó a la prensa madrileña. El Diario Oficial de Avisos de Madrid publicó el 14 de enero la rescisión del contrato del señor Durot, debido a problemas de salud relacionados con sus cuerdas vocales y su sustitución por Francesco Tamagno ${ }^{62}$. El periódico señaló que la baja de Durot le había costado un gran sacrificio económico al conde de Michelena, ya que se había visto obligado a alargar el contrato de Tamagno -en aquella temporada el tenor tenía contratado cantar el Otello deVerdi, Guillermo Tell (1829), Aida (1871) e Il profeta (1849)y a acceder a las exigencias económicas de este.

\section{La complicada adaptación de Puccini en Madrid}

El 16 de febrero de 1892, Puccini le envió a su gran amigo de la infancia, Alfredo Caselli, una carta postal desde Barcelona informándole de su inminente viaje a Madrid para supervisar los ensayos de Edgar ${ }^{63}$. Según el periódico madrileño La Iberia, el maestro italiano llegó a Madrid la mañana del 19 de febrero en el expreso de Barcelona ${ }^{64}$. Debido a que Simoneta Puccini, nieta del compositor, no ha autorizado la publicación de las cartas enviadas entre

\footnotetext{
${ }^{58}$ La carta del tenor aceptando interpretar Edgar no se ha conservado. Puccini agradece a Tamagno su participación en la carta que le escribe desde Milán, el 13-1-1892. Giacomo Puccini. Epistolario I..., p. 181.

59 Carta de G. Puccini a Francesco Tamagno, Milán, 13-1-1892. Giacomo Puccini. Epistolario I..., p. 181.

${ }^{60} \mathrm{Ibid} ., \mathrm{p} .178$.

61 "Grazie tua lettera - Io credo quando avrai letto l'opera comprenderai che da parte tua non avrai molta difficoltà, primo per la facilità tua fenomenale d'apprendere presto, poi per la non difficile parte d'Edgar - A Madrid poi tutto è pronto per l'andata in scena: credo dunque che con 2 o 3 prove al più potresti andare in scena. [...] Intanto ti saluto cordialmente e sperando che questo mio sogno si effettui mi dico tuo aff[ettuoso] G. Puccini". Ibid., p. 181.

62 "Teatros. Real", Diario oficial de avisos de Madrid, 14-1-1892, p. 3.

63 "Da Barcelona 16.2.92 Dove mi fermo sino a Giovedì mattina [18 febrero] per prender il diretto per Madrid il quale c'è 3 voltre alla settimana. Di là ti riscriverò". Carta de G. Puccini a Alfredo Caselli, Barcelona, 16-2-1892. Giacomo Puccini. Epistolario I..., p 186.

64 "Teatro Real", La Iberia, Madrid, 19-2-1892, p. 3.
} 
Puccini y su esposa, Elvira Bonturi, para detallar las primeras impresiones del músico sobre el país y de la ciudad madrileña es necesario basarse en la correspondencia publicada en la biografia de George R. Marek, que tuvo acceso a ese epistolario y cita fragmentos de algunas de esas cartas ${ }^{65}$. Según Marek, Puccini pisó suelo madrileño no el 19, como refirió la prensa, sino el 20 de febrero a las cuatro y media de la tarde ${ }^{66}$.

Durante su estancia madrileña el maestro se alojó en un piso situado en el número 7 de la calle Ferraz, en el barrio madrileño de Argüelles ${ }^{67}$. Cuenta José Subirá que el italiano recibió la visita de figuras representativas de la sociedad madrileña como el novelista Vicente Blasco Ibáñez, el cronista José Gutiérrez Abascal, el crítico wagnerista Félix Borrell o los compositores Ruperto Chapí y Camille Saint-Saëns ${ }^{68}$. Luigi Mancinelli se hospedaba en el número 50 de la misma calle, lo que nos invita a sospechar que el joven maestro alquiló la vivienda en aquella zona por recomendación del director de orquesta, puesto que, en un primer momento, Puccini iba a instalarse en la casa del propio Mancinelli ${ }^{69}$. Además de poder contar con el respaldo del director italiano por la cercanía de sus viviendas, la calle Ferraz estaba próxima al Teatro Real.

El maestro italiano se alojó en una zona muy diferente a la que, por ejemplo, eligió Giuseppe Verdi para su estancia en Madrid con motivo de la representación en el Teatro Real, en el año 1863, de La forza del destino (1862). Parece ser que el vecchio compositor se hospedó en uno de los apartamentos que Luis Nobile de Cataldi alquilaba en el número seis de la Plaza de Oriente ${ }^{70}$. Un complejo residencial donde también solían alojarse los artistas que actuaban en el Real ${ }^{11}$. Es posible que Puccini se instalase en Ferraz por cuestiones económicas, pues en aquel momento era un joven compositor que estaba comenzando su carrera profesional. Lo recaudado con Le Villi y Edgar y las 300 liras mensuales que Ricordi le ingresaba no eran suficientes para hacer frente a los gastos de cada mes ${ }^{72}$.

\footnotetext{
${ }^{65}$ George Richard Marek: Puccini: A Biography, Nueva York, Simon and Schuster, 1951.

${ }^{66}$ Ibid., p. 100.

${ }^{67}$ Actualmente el bloque de viviendas contiene una placa conmemorativa que recuerda su estancia. "Giacomo Puccini", Memoria de Madrid (http://especiales.memoriademadrid.es/index/verficha/idpk/ 58009/id/8/obj/A/idag/25, consulta 20-12-2018).

${ }^{68}$ J. Subirá: "Puccini y el puccinismo en Madrid...", p. 49.

${ }^{69}$ Carta de Luigi Mancinelli a G. Puccini, Madrid, antes del 4 marzo 1891. Luigi Mancinelli. Epistolario..., p. 142

${ }^{70}$ Víctor Sánchez: Verdi y España. Madrid, Ediciones Akal, 2014, p. 179.

${ }^{71}$ Ibid.

${ }^{72}$ Ibid.
} 
Aun así, el barrio que hospedó a Puccini durante su mes en Madrid fue una zona elegante y burguesa en la que vivían familias de elevado poder adquisiti$\mathrm{vo}^{73}$. En ella se construyeron hoteles rodeados de jardines, viviendas unifamiliares y pisos de alquiler ${ }^{74}$. De hecho, la zona en la que el músico se instaló (entre la calle Rey Francisco y calle Tutor) fue destinada a casas de alquiler ${ }^{75}$. También se abrieron comercios de primera necesidad (droguerías, farmacias, tiendas de tejidos, etc.) y edificios destinados al entretenimiento como locales de espectáculos, terrazas y cafeterías ${ }^{76}$.

Durante su estancia es posible que Puccini asistiera a algunas de las obras que se estaban representando en los escenarios musicales de la ciudad. El mismo Saint-Saëns (1835-1921) era un asiduo visitante de los teatros de género chico cuando viajaba a Madrid, por lo que Puccini pudo hacer lo mismo. Si rastreamos la cartelera madrileña durante ese mes, el compositor podría haber asistido a la representación de zarzuelas como Tiquis Miquis $(1883)^{77}$ del maestro Ruperto Chapí (1851-1909), con el cual parece ser que mantuvo muy buena relación durante su estancia ${ }^{78}$; haber escuchado obras de Federico Chueca (1846-1908) como De la noche a la mañana $(1883)^{79}$, ¡A los toros! (1877) ${ }^{80}$ o La caza del oso o El tendero de comestibles $(1891)^{81}$; o haber asistido a algunas de las audiciones de la Sociedad de Conciertos que Luigi Mancinelli dirigía en el Teatro del Príncipe Alfonso $^{82}$. También pudo participar en las tertulias musicales que tenían lugar en la Casa Lhardy y entrar en contacto con otros compositores españoles, así como con los artistas, políticos o periodistas que lo frecuentaban. Entre los componentes de este círculo madrileño destacan críticos y musicólogos como Peña y Goñi, Cecilio de Roda, Joaquín Fesser, los hermanos Félix y José Borrell o Alejandro Saint-Aubin ${ }^{83}$, los cuales serán los responsables de juzgar los estrenos de las óperas puccinianas en Madrid. La Casa Lhardy fue el centro de tertulias wagnerianas más popular de la ciudad a finales de si-

\footnotetext{
${ }^{73}$ Alicia Díez de Baldeón García: "El nacimiento de un barrio burgués. Argüelles en el siglo XIX", Norba: Revista de Arte, 13, 1993, p. 231.

${ }^{74}$ Ibid., p. 268.

${ }^{75} \mathrm{Ibid}$., p. 245.

${ }^{76}$ Ibid., p. 267.

77 "Espectáculos para mañana. Lara", La Época, Madrid, 25-2-1892, p. 4.

${ }^{78}$ Luis G. Iberni: Ruperto Chapí, Madrid, ICCMU, 1995, p. 306.

79 "Espectáculos para mañana. Apolo", La Correspondencia de España, Madrid, 5-3-1892, p. 4.

80 "Espectáculos para el día 7. Princesa", La Correspondencia de España, Madrid, 7-3-1892, p. 3.

81 "Espectáculos para el día 10. Novedades", La Correspondencia de España, Madrid, 10-3-1892, p. 4.

82 "Espectáculos para mañana. Príncipe Alfonso", La Correspondencia de España, Madrid, 6-3-1892, p. 4.

83 Paloma Ortiz: "La recepción de Richard Wagner en Madrid (1900-1914)", Cuadernos de Música Iberoamericana, 12, pp. 246-247.
} 
glo XIX y principios del XX. Constituyó el núcleo oficial de las reuniones de los aficionados a la obra del compositor alemán entre los que se encontraba Luigi Mancinelli ${ }^{84}$.

Políticamente, la llegada de Puccini coincide con la Restauración y el desarrollo de la política canovista. Tras los convulsos años del Sexenio Revolucionario, el país vivió una época de calma y de cierta prosperidad económica que permitió a una emergente clase burguesa frecuentar los espectáculos.

Los primeros días en la ciudad fueron complicados para el maestro. Las dificultades para entender el español y el encontrarse solo en una ciudad extranjera, rodeado de gente desconocida, hizo más costosa su adaptación en el país tal y como lo refleja la siguiente carta escrita a su mujer Elvira (todavía casada con el farmacéutico de Lucca, Narciso Gemignani) ${ }^{85}$ el 23 de febrero de 1892:

Pobre Topizio, estoy solo, solo sin mi Topizia. ¡Cómo pienso en ti, mi ángel! ¡Cómo deseo tenerte cerca de mí! Daría diez años de mi vida por tenerte aquí. [...]. Estoy cansado de Madrid y de este país tan poco comprensivo, donde se vive de forma tan diferente a como se vive en $\operatorname{casa}^{86}$.

La soledad, su desconocimiento del idioma y los continuos problemas para estrenar Edgar dieron lugar a que el italiano considerase Madrid como una ciudad "odiosa" 87 . Además, el músico había llegado con una imagen idealizada de España. En una carta escrita a su mujer, Puccini da a entender que previamente a su viaje a Madrid conocía o había leído el libro del escritor Edmondo de Amicis, España: viaje durante el reinado de Don Amadeo I (1883) ${ }^{88}$. En esa carta, el maestro expresó su malestar con el autor por haber escrito "tanta poesía sobre este miserable y presuntuoso país" 89 y haber dado una imagen que no se ajustaba con la realidad que él estaba viviendo, lo que provocó que "tan pronto como llegue a Milán, compraré su libro para ponerlo en la letrina" ${ }^{90}$. Edmondo de Amicis describió un Madrid de calles limpias y espaciosas con numerosos cafés, salas de espectáculos y casas de vivos colores, así como, repleto de gente elegante, alegre y

\footnotetext{
${ }^{84}$ Ibid., p. 247.

${ }^{85}$ Mary Jane Phillips-Matz: Puccini: A Biography, Boston, Northeastern University Press, 2002, p. 48.

86 "Poor Topizio, I am alone, alone without my Topizia. How I think of you, my angel! How I desire to have you near me! I'd give ten years of my life to have yo here. [...] I am tired of Madrid and of this unsympatic country, where one lives so differently from at home [...]". G. R. Marek: Puccini: A Biography..., p. 100.

${ }^{87}$ Carta de Giacomo Puccini a Elvira Bonturi, Madrid, 16-3-1892; G. R. Marek: Puccini: A Biography..., p. 101.

${ }^{88}$ Edmondo de Amicis: España: viaje durante el reinado de Don Amadeo I, Traducido por Augusto Suárez de Figeroa, Madrid, Librería de Vicente López, 1883.

89 "Who has written so much poetry about this miserable and presumptuous country". G. R. Marek: Puccini: A Biography..., Ibid.

90 "As soon as I get to Milan, I will buy his book in order to put it in the latrine". G. R. Marek: Puccini: A Biography... Ibid.
} 
acogedora ${ }^{91}$.Acerca del Teatro Real (Teatro de la Ópera), De Amicis lo consideró como un edificio magnífico arquitectónicamente y resaltó que el pueblo español era muy aficionado al género de la ópera italiana ${ }^{92}$.

La incomodidad de esos primeros días se acrecentó con los ensayos de Edgar. Menciona Marek que la noche del 20 de febrero, Puccini asistió al Teatro Real para escuchar a Tamagno cantar el Guillermo Tel ${ }^{93}$, quedando sorprendido por el gran nivel del coro y de la orquesta del teatro. No obstante, pronto se esfumó tal ilusión y Puccini le comunicaba a Ricordi que, en los ensayos de Edgar, "los coros son discretos y la orquesta igual -no hacen nada más y las notas no tienen fuerza-, todos parecen tocar y cantar bajo el escenario" "94. Aunque el maestro italiano se mostró contento con los ensayos realizados, la lentitud de estos y el poco compromiso mostrado por el coro y la orquesta le hicieron desilusionarse.

Además, Puccini tuvo que hacer frente a otros acontecimientos que aceleraron su deseo de abandonar Madrid. En primer lugar, el maestro tuvo que lidiar con algunos problemas relacionados con los cantantes y que estaban afectando al buen rendimiento de la ópera. La mezzosoprano italiana Giuseppina Pasqua, quien encarnaba el papel de Tigrana, mostró signos de cansancio en la voz durante los ensayos, peligrando su participación para el día del estreno ${ }^{95}$. En cuanto a Tamagno, quien según el compositor estaba espléndido en el papel de Edgar, a Puccini no le agradaron las discusiones entre este y el conde de Michelena debido a las exigencias económicas que pedía el tenor a escasos días del estreno. Tamagno había amenazado a la dirección del Teatro Real con abandonar Edgar si esta no ampliaba su contrato por dos actuaciones más ${ }^{96}$, lo que provocó en Puccini una gran decepción hacia el tenor. Tamagno ponía así en peligro la representación de la ópera por una cuestión de egoísmo económico.

Por otro lado, al maestro italiano le dolieron los comentarios recibidos por compositores españoles como Emilio Serrano y Tomás Bretón (1850-1923): “Ayer salió un periódico, 'La Correspondencia', diciendo pestes del libreto y esto es muy malo siendo dicho periódico muy leído. Serrano, el Maestro, me

\footnotetext{
${ }^{91}$ Edmondo de Amicis: España: viaje durante el reinado de Don Amadeo I..., p. 129.

${ }^{92}$ Edmondo de Amicis, Op. cit., p. 143.

${ }^{93}$ Suponemos que Puccini asistiría a uno de los ensayos generales ya que Guillermo Tell se estrena el 23 de febrero. Véase Joaquín Turina: Historia del Teatro Real..., p. 409.

94 "I cori son discreti e l'orchestra idem - fanno niente di più e le note non hanno forza - par che suonino, e cantino tutti sotto il palco". Carta de G. Puccini a Giulio Ricordi, Madrid, 8-3-1892. Giacomo Puccini. Epistolario I..., p. 188.

${ }^{95}$ Carta de G. Puccini a Giulio Ricordi, Madrid, 8-3-1892. Giacomo Puccini. Epistolario I..., p. 188.

${ }^{96}$ Ibid.
} 
hace una cruzada atroz en contra y Bretón me dicen que también"97. La carta en la cual Puccini le informa a Ricordi de dichos comentarios fue escrita el día 8 de marzo de 1892. Efectivamente, La Correspondencia de España, publicó ese día un artículo bastante negativo sobre el argumento ${ }^{98}$. En cuanto a los comentarios realizados por Emilio Serrano y Tomás Bretón es muy probable que se hubiesen realizado en tertulias o en los saloncillos de los teatros de zarzuela.

El único rasgo positivo que destacó el compositor de esta producción madrileña fueron las admirables decoraciones realizadas por el escenógrafo italiano Giorgio Busato ${ }^{99}$. El pintor escenógrafo del Teatro Real, nacido enVicenza, había llegado al teatro madrileño en el año 1887. Fue un gran colorista y se caracterizó por su incomparable tratamiento de las arquitecturas escénicas ${ }^{100}$.

De forma previa al estreno, la prensa madrileña presentó al compositor, trazando su perfil biográfico y artístico, así como los rasgos más característicos de la partitura y un resumen del libreto. El maestro es en estos momentos en España un completo desconocido. Desde el punto de vista de la recepción, a través de estas noticias sabemos qué aspectos de la vida del músico fueron conocidos por los madrileños. De Puccini, se señaló que era un joven compositor de treinta y tres años que pertenecía a una dinastía de músicos y cuyo comienzo había estado marcado por apuros económicos, pero que contaba con un "mecenas incomparable: Ricordi" "101. Cuando Puccini llegó a España y pudo entablar contacto con las personalidades del momento, el compositor fue descrito como un hombre ilustrado, de gran ingenio, que hablaba poco pero que escuchaba mucho y atentamente ${ }^{102}$. Sin embargo, otro diario lo describía como "un hombre muy ilustrado, habla con viveza, su conversación es chispeante, tiene gran ingenio y es oriundo de la Toscana, esa cuna de tantos eminentes artistas" ${ }^{\prime 103}$. Sobre su manera de componer, los periódicos madrileños publicaron que:

La música de Puccini tiene un sello característico de originalidad, y revela que, sin desdeñar los procedimientos modernos en cuanto a instrumentación se refiere, el autor huye del plagio, no reniega de la tradición italiana y tiene procedimientos propios, personalísimos, unidos a rasgos de verdadero genio [...]. Buena o mala, la música de Puccini es siempre suya ${ }^{104}$.

97 "Ieri è uscito un giornale 'La Correspondencia' dicendo ira dei del libretto e questo è molto male essendo detto giornale molto letto - Serrano il Maestro mi fa una crociata atroce contro e così mi dicono anche Bretón". Ibid.

98 "Edgar", La Correspondencia de España, Madrid, 8-3-1892, p. 2.

${ }^{99}$ Carta de G. Puccini a Giulio Ricordi, Madrid, 8-3-1892. Giacomo Puccini. Epistolario I..., p. 188.

${ }^{100}$ Juan Paz Canalejo: La caja de las magias: las escenografias históricas en el Teatro Real, Madrid, Ayuntamiento de Madrid, 2006, p. 174.

${ }^{101}$ Antonio Peña y Goñi: “Crónicas madrileñas. El autor de 'Edgar”, La Época, Madrid, 17-3-1892, p. 2.

102 Ibid.

${ }^{103}$ Allegro: "Edgar. El compositor", El País, Madrid, 7-3-1892, p. 2.

104 "Puccini y su ópera Edgar", El Día, Madrid, 19-3-1892, p. 1. 
No obstante, en los próximos estrenos puccinianos en el país, el estilo ecléctico de Puccini fue señalado como un rasgo negativo en su música y utilizado como discurso para minusvalorar su producción. Igualmente ocurrió con la supuesta influencia de Wagner en su manera de componer, ya que le hacía parecer sospechoso de carecer de personalidad ${ }^{105}$.

\section{El estreno de Edgar en el Teatro Real}

El estreno de Edgar se verificó en el Teatro Real el 19 de marzo de $1892^{106}$. Los periódicos señalaron que la expectación ante el estreno era más que evidente por las buenas impresiones recogidas en el ensayo general ${ }^{107}$ y porque la ópera constituía la única novedad musical de la temporada ${ }^{108}$. A juzgar por los aplausos y las llamadas a escenas del maestro y los $\operatorname{artistas}^{109}$, la prensa señaló que la obra obtuvo una acogida favorable por parte del público. Entre los asistentes se encontraron la reina regente María Cristina, la cual parece ser que llamó al maestro a su palco para felicitarle ${ }^{110}$, y la infanta doña Isabel (La Chata) a la que Puccini le dedicó, tras su regreso a Milán, una fotografia suya ${ }^{111}$.

$\mathrm{Al}$ analizar las críticas, se observa una fractura en la recepción del músico entre aquellos que continuaban abogando por la ópera italiana y los que se oponían a la hasta entonces omnipresente tradición y que, por lo tanto, eran partidarios de las nuevas tendencias germanas, concretamente de Wagner. Antonio Peña y Goñi ${ }^{112}$, caracterizado por un wagnerismo fervoroso, daba fe de la buena acogida del italiano en Madrid y le daba la bienvenida a nuestro país ${ }^{113}$. Una opinión bastante contraria al crítico de El País Pascual Millán -quien firmaba bajo el seudónimo Allegro-, que también era wagneriano. Allegro, quien se convertiría en uno de los grandes detractores del maestro en Madrid, cuestionaba que la obra fuese "digna de la acogida que obtuvo"114. Edgar se estrena en un momento de polaridad extrema entre wagnerianos y partidarios

\footnotetext{
${ }^{105}$ Allegro: "En el teatro de la Ópera. Manon Lescaut", El País, Madrid, 5-11-1893, p. 2.

106 "Puccini y su ópera Edgar", El Día, Madrid, 19-3-1892, p. 1.

${ }^{107}$ Ibid.

${ }^{108}$ Antonio Peña y Goñi, "El autor de 'Edgar”, La Época, Madrid, 17-3-1892, p. 1.

109 "Desde mi butaca. Teatro Real. Edgar", El Día, Madrid, 20-3-1892, p. 2.

${ }^{110}$ Julian Budden: Puccini..., 2007, p. 84.

${ }^{111}$ Puccini escribió en la dedicatoria: "A sua Altezza Reale Doña Ysabella de Borbón. Contessa di Girgenti. [...] melte offre G. Puccini. Milano 27.03.92". Gaspar Gómez de la Serna: Gracias y desgracias del Teatro Real (Abreviaturas de su historia), Madrid, Servicio de Publicaciones del Ministerio de Educación y Ciencia, 1976, p. 54.

${ }^{112}$ Rubén Corchete Martínez: Antonio Peña y Goñi (1846-1896). La institucionalización de la crítica musical decimonónica, trabajo final de máster, Universidad de Oviedo, 2014

${ }^{113}$ Antonio Peña y Goñi: "Teatro Real. Edgar", La Época, Madrid, 20-3-1892, p. 1.

${ }^{114}$ Allegro: "En el Teatro de la Ópera. Edgar", El País, Madrid, 20-3-1892, p. 1.
} 
de la ópera italiana. Esta rivalidad será una cuestión constante en la recepción del músico en la capital española. Respecto al tema, el crítico Joaquín Arimón hacía las siguientes declaraciones tras el estreno:

Habría sido preferible poner en escena El buque fantasma, de Wagner, tan pomposamente anunciado en el cartel de abono. Pero la empresa del teatro Real ha pensado de otro modo, y nos ha ofrecido en cambio una ópera de autor novel, pero eficazmente recomendada por la famosa casa Ricordi de Milán, a la que pocos ganan en materia de habilidad para la propagación de sus fondos editoriales por todo el orbe musical. En España contamos con compositores que, cuando menos, están a la altura de Puccini, y bien pudiera estimulárseles con preferencia a los maestros extranjeros, a quienes no abona en absoluto la supremacía del genio ${ }^{115}$.

El supuesto poder que la Casa Ricordi ejercía sobre la programación del Teatro Real no solo afectó a la ópera wagneriana sino también a la ópera nacional. El crítico del diario La Justicia, R. Melgrán, hizo públicas sus quejas sobre la ausencia de óperas de autores españoles en la programación del teatro a pesar de la obligación de la empresa de representarlas: ¿QQuién ignora que el Teatro Real no es más que una sucursal de la Casa Ricordi! ¡Quién desconoce que el poderoso editor italiano nos impuso el Simón Boccanegra, de Verdi, y el Edgar de Puccini!"116. El panorama operístico en España en aquel momento era variado. Las obras del compositor alemán empezaban a asentarse en la capital española gracias a la labor de Mancinellii17, el repertorio italiano copaba buena parte de la práctica musical de la época y el debate en torno a la creación de la ópera nacional volvía a reabrirse.

Para la mayoría de los críticos Edgar no era una obra maestra y, como trabajo de juventud, contenía defectos propios de un compositor en formación, a la vez que se destacaban ya en la partitura destellos que anunciaban el sobresaliente porvenir del músico ${ }^{118}$. La brillantez de las melodías, característica hoy indiscutible de su estilo, fue percibida ya por la crítica como la influencia de "la musa del arte patrio"119. Según las críticas publicadas, el legado de la escuela italiana se hacía patente en la claridad melódica y en su habilidad para describir y expresar a través de ella los sentimientos de los personajes. Como ejemplo de ello, la prensa madrileña señalaba el acto tercero, que se correspondía con el momento de dolor de Fidelia en la escena del funeral de Edgar, donde "aquí siente Puccini como sienten los poetas y expresa como expresan los artistas

\footnotetext{
115 Joaquín Arimón: “Teatro Real. 'Edgar”, El Liberal, Madrid, 20-3-1892.

${ }^{116}$ R. Melgrán: "Revista Musical. La próxima temporada del teatro de la Ópera", La Justicia, Madrid, 17-7-1893, p. 1 .

117 J. I. Suárez: "La recepción de la obra de Richard Wagner...", pp. 102-122.

118 "Desde mi butaca. Teatro Real. Edgar", El Día, Madrid, 20-3-1892, p. 2.

119 Ricardo González: "Teatro Real. Manon Lescaut", La Correspondencia de España, Madrid, 6-11-1893, p. 4.
} 
verdaderos y siente además y expresa como italiano que es" ${ }^{120}$. En definitiva, para los críticos madrileños, en Edgar, Puccini se debatía entre la influencia de la escuela italiana, de donde aprendió el oficio, y la herencia wagneriana de la que no pudo escapar como artista joven que era, interesado en las tendencias musicales en boga.

Aparte de Francesco Tamagno, la representación corrió a cargo del barítono Ignacio Tabuyo (Frank), la soprano Eva Tetrazzini (Fidelia) y Giuseppina Pasqua (Tigrana). Sobre los artistas, la crítica lanzó elogios por su ejecución destacándose especialmente la de Tagmano, que fue considerado "el alma de la representación": "Si otro tenor cualquiera de los actuales canta la ópera, muchas de las frases aplaudidas anoche hubieran pasado en silencio" "121. La presencia de Tamagno demuestra hasta qué punto el éxito de un estreno no dependía de las obras en sí mismas, pues el tenor condicionó cierto ambiente favorable hacia la ópera.

Los números musicales más aplaudidos por el público madrileño fueron los dos primeros actos de la ópera, concretamente el preludio, el aria de Fidelia y el concertante final del primer acto; la romanza de Edgar y el dueto con Tigrana del segundo acto; y, del último, el terceto interpretado por Giuseppina Pasqua, Tamagno y Tabuyo ${ }^{122}$. Los críticos señalaron que el mayor triunfo de Puccini en el estreno había sido que el público pidiera la repetición de algunos de los números de la ópera. Según el diario El Día, el público del Teatro Real no era nada fácil puesto que, solía escuchar con desconfianza los estrenos de las obras a pesar de pertenecer a grandes maestros y ser famosas en Europa ${ }^{123}$. Para Peña y Goñi:"El público se hizo amigo de Puccini, pero amigo de verdad, interesado en el porvenir del maestro y dispuesto a recibirlo con simpatía cuantas veces llame a las puertas del Teatro Real"124.

En la prensa madrileña se volvieron a repetir algunos de los comentarios que el compositor y la ópera ya habían recibido por parte de sus compatriotas. El libreto fue señalado nuevamente como el "defecto capital de la obra"125, ya que ni interesaba, ni emocionaba, ni era verosímil. Para los críticos madrileños, la sensación que se percibió en el teatro fue la de unos personajes que parecían “autómatas que cantaban dúos, romanzas y concertantes [donde] el público no veía en ellos a los héroes y heroínas de la fábula sino a Tamagno, a Tabuyo, a la Tetrazzini y a la Pascua"126. Por suerte, los críticos supieron apreciar que los

\footnotetext{
120 Antonio Peña y Goñi: "Teatro Real. Edgar", La Época, Madrid, 20-3-1892, p. 1.

${ }^{121}$ Allegro: "En el Teatro de la Ópera. Edgar", El País, Madrid, 20-3-1892, p. 1.

${ }^{122}$ D. Díaz González: "La crítica wagneriana...", p. 158.

123 "Desde mi butaca. Teatro Real. Edgar", El Día, Madrid, 20-3-1892, p. 2.

${ }^{124}$ Antonio Peña y Goñi: "Teatro Real. Edgar", La Época, Madrid, 20-3-1892, p. 1.

${ }^{125}$ Ricardo González: "Teatro Real. Manon Lescaut", La Correspondencia de España, Madrid, 6-11-1893, p. 4.

${ }^{126}$ Ibid.
} 
defectos en la música no eran causados solamente por falta de experiencia en el músico, sino que la "vulgaridad" 127 del libreto había hecho mella en la buena recepción de la ópera.

Otro de los comentarios que el maestro recibió en Italia fue que su música resultaba demasiado ruidosa. En Madrid, la respuesta a por qué la partitura de Edgar sonaba tan estridente se encontró en el libreto. Para los críticos madrileños, Puccini, al intentar no ser arrastrado por "uno de tantos argumentos de ópera" 128 , cayó en lo artificioso y en el exceso, reduciendo la voz y agrandando la orquesta mediante el empleo de grandes masas corales y de todos los instrumentos ${ }^{129}$. Con ello, el efecto conseguido fue el de "aturdir y acabar cubriendo las delicadas melodías de la ópera"130. Por tanto, ese afán que mostraba el compositor por que toda la orquesta sonara debía ser corregido, pues reconocían en él a un gran orquestador ${ }^{131}$.

La dirección musical y la puesta en escena fueron muy bien valoradas. Sobre todo esta última, cuyos decorados, realizados por Giorgio Busato y Bernardo Bonardi, recibieron una ovación tanto por el público como por la crítica.Tal es así que Allegro aseguraba al maestro Puccini que "no volverá a oírla mejor interpretada ni a verla con más esplendor" 132 .

Tras finalizar las tres funciones, Puccini abandonó Madrid al día siguiente (23 de marzo), tal y como demuestra una carta escrita por el músico a Francesco Tamagno. En ella, Puccini le agradecía su sobresaliente interpretación al mismo tiempo que le pedía disculpas por no haberse podido despedir de él tras la última representación ${ }^{133}$.

\section{Conclusiones}

La ópera se representó durante tres noches y aunque su acogida fue más favorable que en Italia, el estreno en Madrid no fue suficiente para que la obra resurgiera de su fracaso. A pesar de que Edgar es la ópera menos representada de las obras de Puccini y que el Teatro Real no ha vuelto a programarla, es innegable que marca un momento importante en la recepción del músico en Madrid. Esta producción madrileña supuso la primera visita de Puccini a un país extranjero para supervisar los ensayos de sus óperas, la primera producción de Edgar fuera de Italia y el único viaje del italiano a España.Aunque el maestro se marchó con la satisfacción de que la crítica y el público fueron benévolos

\footnotetext{
${ }^{127}$ Antonio Peña y Goñi: "Teatro Real. Edgar", La Época, Madrid, 20-3-1892, p. 1.

128 Allegro: "Edgar. El compositor", El País, Madrid, 7-3-1892, p. 2.

${ }^{129}$ Antonio Peña y Goñi: "Teatro Real. Edgar", La Época, Madrid, 20-3-1892, p. 1.

130 "Desde mi butaca. Teatro Real. Edgar", El Día, Madrid, 20-3-1892, p. 2.

${ }^{131}$ Ibid.

${ }^{132}$ Allegro: "En el Teatro de la Ópera. Edgar", El País, Madrid, 20-3-1892, p. 2.

133 Ugo Piovano: Otello Fu, Milán, Rugginenti Editore, 2005, p. 341.
} 
con él, se llevó una mala impresión del país. A través de la correspondencia del músico se ha podido ver que la soledad durante su estancia, su desconocimiento del idioma y los continuos problemas para estrenar la obra hicieron que el italiano se llevara una negativa imagen de la ciudad y del estereotipo de los españoles. Además, su incomodidad pudo haberse acrecentado por la previa lectura del libro de Edmondo de Amicis, en la que el escritor mostraba una visión sobre el país completamente distinta a la vivida por Puccini. Estos pudieron ser los motivos que propiciaron que el italiano no regresara en otra ocasión a España.

Por otro lado, debemos destacar el papel del director de orquesta Luigi Mancinelli, pues sin su presencia en el teatro hubiera sido casi imposible que Edgar se llegase a estrenar en Madrid. El director italiano fue el medio de conexión entre la empresa del Real y la Casa Ricordi y el que intermedió en las negociaciones de ambos.

En cuanto a la recepción del músico, Puccini fue visto como un joven compositor prometedor que compartía cartel junto a los grandes maestros. De todos ellos, las comparaciones con Wagner serían inevitables tras la huella que el alemán dejó en la ciudad. La postura de los críticos wagnerianos fue ambigua pues, mientras Peña y Goñi daba la bienvenida al compositor italiano y lo felicitaba por su triunfo, Allegro veía en él a un maestro que progresaba merced a un poderoso editor. También se observa desde este momento una fractura en la recepción del músico entre aquellos que rechazan la hasta entonces omnipresente escuela italiana (wagnerianos y defensores de la ópera nacional) $-\mathrm{y}$ que, por lo tanto, denigran al músico-, y los que defienden a Puccini por continuar abogando por la tradición de su país y ofrecer un género muy arraigado en España: la ópera italiana.

También fue importante la presencia del tenor Francesco Tamagno en la representación de la ópera, pues la buena acogida de la obra dependió en parte de su participación debido a la gran fama del cantante tras su interpretación como Otello. De este modo, el aparato productivo y empresarial de Edgar (Ricordi-Mancinelli-Tamagno) fue fundamental en la recepción del primer Puccini en España.

Recibido: 5-3-2019

Aceptado: 29-8-2019 